\title{
ON THE COMPLETE INTEGRABILITY OF NONLINEAR PARTIAL DIFFERENTIAL EQUATIONS
}

\author{
BY M. S. BERGER ${ }^{1}$ AND P. T. CHURCH
}

The concept of complete integrability for nonlinear Hamiltonian systems of finite dimension $2 N$ is generally based on a theorem of Liouville (cf. [1, p. 271]), that requires the Hamiltonian system to possess $N$ independent first integrals in involution. Recently this notion has been extended to infinite-dimensional Hamiltonian systems by a number of authors (for example, Faddeev and Zacharov, Gardner, Lax, McKean, Novikov, Gelfand and Dikii, among others) who have shown that certain nonlinear partial differential equations in two dimensions are integrable in this sense of Liouville provided one lets $N \rightarrow \infty$. In particular the celebrated Korteweg-de Vries

$$
u_{t}+u u_{x}+u_{x x x}=0
$$

is completely integrable in this sense.

However, this notion of complete integrability seems to be of limited value for treating nonlinear partial differential equations in more than two variables. Moreover, the study of the perturbations of a system completely integrable in this sense of Liouville generally requires radically new methods, since the first integrals (whose existence is intrinsic to the Liouville approach) are generally destroyed.

In this article we define a new type of complete integrability for nonlinear elliptic boundary value problems (in fact for nonlinear mappings between Banach spaces), and show, by example, that an explicit nonlinear Dirichlet problem $\left(\pi_{n}\right)$ defined on an arbitrary bounded domain $\Omega \subset \mathbf{R}^{n}$ with dimension $n$ arbitrary is completely integrable in our sense. Moreover, our methods of study are sufficiently flexible to yield significant results for a $C^{1}$ perturbation $\tilde{\pi}_{n}$ of $\pi_{n}$.

1. The notion of complete integrability. Let $A$ denote a given smooth (say $C^{k+1}$ ) mapping between two real Banach spaces $X_{1}, X_{2}$. Then we say $A$ is globally $C^{k}$ equivalent to a mapping $B$ between $X_{3}$ and $X_{4}$ if there are $C^{k}$ diffeomorphisms $\alpha$ and $\beta$ such that the following diagram commutes

Received by the editors March 6, 1979.

AMS (MOS) subject classifications (1970). Primary 39A15; Secondary 47D20.

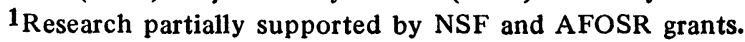

๑ 1979 American Mathematical Society 0002-9904/79/0000-0321/\$01.75 


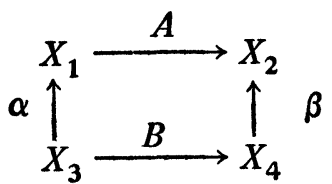

so that $A \alpha(x)=\beta B(x)$ for each $x \in X_{3}$.

The simplest case occurs when $X_{1}=X_{2}=X_{3}=X_{4}$ are the same separable Hilbert space; then [relative to an orthonormal basis $\left.\left(x_{1}, x_{2}, x_{3}, \ldots\right)\right] . A$ can be regarded as the coordinate mapping

$$
A\left(x_{1}, x_{2}, x_{3}, \ldots\right)=\left(A_{1}, A_{2}, A_{3}, \ldots\right), \text { with } A_{i}=\left(A, x_{i}\right) x_{i}
$$

The mapping $A$ will then be called $C^{k}$ completely integrable if there is a mapping $B$ of the form

$$
B\left(x_{1}, x_{2}, x_{3}, \ldots\right)=\left(g_{1}\left(x_{1}\right), g_{2}\left(x_{2}\right), g_{3}\left(x_{3}\right), \ldots\right)
$$

where the real-valued function $g_{i}\left(x_{i}\right)$ is a smooth function of $x_{i}(i=1,2,3, \ldots)$ such that $A$ and $B$ are globally $C^{k}$ equivalent in the sense defined above. (Thus $B$ "diagonalizes" $A$ and links the gradient nature of $A$ to Hilbert space theory.) More generally for distinct real separable Banach spaces $X_{i}(i=1,2,3,4)$ with basis the equation (3) also provides a natural notion of global $C^{k}$ equivalence.

2. The nonlinear boundary value problem. Consider the following nonlinear elliptic Dirichlet problem studied in ([2], [3], [4])

$$
\left\{\begin{array}{r}
\Delta u+f(u)=g \\
\left.u\right|_{\partial \Omega}=0
\end{array}\right.
$$

defined on a bounded domain $\Omega \subset \mathbf{R}^{n}$, with boundary $\partial \Omega$. Here $f(t)$ is a $C^{j}$ $(j \geqslant 2)$ real-valued domain function with $f^{\prime \prime}(0)>0$ satisfying the asymptotic conditions

$$
0<\lim _{t \rightarrow-\infty}\left(\frac{f(t)}{t}\right)<\lambda_{1}<\lim _{t \rightarrow \infty}\left(\frac{f(t)}{t}\right)<\lambda_{2}
$$

where $\lambda_{1}$ and $\lambda_{2}$ denote the lowest two eigenvalues of the Laplace operator $\Delta$ relative to null Dirichlet boundary conditions.

To relate $\pi_{n}$ to the notion of complete integrability described above we define a mapping $A$ of the Sobolev space $H=\stackrel{\circ}{W}_{1,2}(\Omega)$ into itself by setting

$$
(A u, \phi)_{H}=\int_{\Omega}(\nabla u \cdot \nabla \phi-f(u) \phi) d x
$$


If $j=2, A$ is easily shown to be a $C^{1}$ mapping of $H$ into itself ([3]). For $j \geqslant 3$, it is convenient to consider a mapping $\mathscr{Q}=\Delta u+f(u)$ between the Hölder spaces $C^{2, \alpha}(\Omega)$ and $C^{0, \alpha}(\Omega)$ (with $0<\alpha<1$ ). Then we can prove

TheOREM 1. For $j=2, A$ [as defined in (4)] is $C^{0}$ completely integrable in the sense that there are canonical homeomorphisms such that $A$ is $C^{0}$ globally equivalent to $B: H \rightarrow H$ defined by

$$
B\left(x_{1}, x_{2}, x_{3}, \ldots\right)=\left(x_{1}^{2}, x_{2}, x_{3}, \ldots\right)
$$

relative to an orthonormal basis of normalized eigenfunctions of $\Delta$. For $j=3$ the operator $\mathfrak{A}$ as defined above is $C^{1}$ completely integrable with the same canonical homeomorphisms relative to the orthonormal basis mentioned above, and $\mathfrak{Q}$ is $C^{1}$ globally equivalent to the operator $B: C^{2, \alpha}(\Omega) \rightarrow C^{0, \alpha}(\Omega)$ defined by $B\left(x_{1}, x_{2}, x_{3}, \ldots\right)=\left(x_{1}^{2}, x_{2}, x_{3}, \ldots\right)$.

As corollaries we find (i) the operators $A$ and $\mathfrak{Q}$ are proper mappings, (ii) for $g \in L_{2}$, the solutions of $\pi_{n}$ can be explicitly determined in terms of the canonical coordinates mentioned in Theorem 1 , (iii) $\pi_{n}$ has either 0,1 or 2 solutions depending on whether or not $g$ is a singular value of the mapping $A$, and (iv) all the singular points are "infinite-dimensional folds" (in the sense of Whitney). Also, (v) consider a sufficiently small $C^{1}$ perturbation $\tilde{f}$ of $f$ in $\left(\pi_{n}\right)$ that destroys the convexity of $f$ and hence the $C^{0}$ complete integrability of $A$; then, using the methods of proof of Theorem 1 , and assuming $\widetilde{f}^{\prime \prime}$ is uniformly bounded, one shows the perturbed Dirchlet problem $\left(\tilde{\pi}_{n}\right)$ and $\left(\pi_{n}\right)$, for $g$ outside a small neighbourhood of the singular values of $A$, have the same number and approximate size of solutions.

Sketch of the proof of Theorem 1. The proof divides into two distinct parts. First an analytical part, in which one determines explicit cartesian representations of the singular points and singular values for the abstract mapping $A$. This is achieved by noting that the mapping $A$ is a diffeomorphism when restricted to the complement of $\operatorname{Ker}\left(\Delta+\lambda_{1}\right)$ and then carefully analyzing the resulting one-dimensional problem so as to prove the operator $A$ is a proper mapping.

The second part of the proof is geometric and consists in constructing canonical coordinate transformations carrying the singular points of $A$ into the singular points of the global fold map $B$, and similarly for the singular values. The same scheme but with somewhat different estimates in the analytical part yields the stated result for the operator 2 .

\section{BIBLIOGRAPHY}

1. V. Arnold, Mathematical methods of classical mechanics, Springer-Verlag, New York, 1978. 
2. A. Ambrosetti and G. Prodi, On the inversion of some differentiable mappings with singularities, Ann. of Math. 93 (1972), 231-246.

3. M. Berger and E. Podolak, On the solutions of a nonlinear Dirichlet problem, Indiana J. Math. 24 (1975), 827-846.

4. L. Nirenberg, Nonlinear functional analysis, New York University, lecture notes, 1974.

SCHOOL OF MATHEMATICS, INSTITUTE FOR ADVANCED STUDY, PRINCETON, NEW JERSEY 08540

DEPARTMENT OF MATHEMATICS AND STATISTICS, UNIVERSITY OF MASSACHUSETTS, AMHERST, MASSACHUSETTS 01003

DEPARTMENT OF MATHEMATICS, SYRACUSE UNIVERSITY, SYRACUSE, NEW YORK 13210 